\title{
STRONG CONVERGENCE OF A HYBRID METHOD FOR INFINITE FAMILY OF NONEXPANSIVE MAPPING AND VARIATIONAL INEQUALITY
}

\author{
Savita Rathee ${ }^{1}$, Monika Swami ${ }^{2}$ \\ Maharshi Dayanand University, Rohtak, 124001, India, \\ ${ }^{1}$ dr.savitarathee@gmail.com, ${ }^{2}$ monikaswami06@gmail.com
}

\begin{abstract}
The motivation behind this paper is to use hybrid method for searching a typical component of the set of fixed points of an infinite family of nonexpansive mappings and the set of monotone, Lipschtiz continuous variational inequality problem. The contemplated method is combination of two method one is extragradient method and the other one is DQ method. Also, we demonstrate the strong convergence of the designed iterative technique, under some warm conditions.
\end{abstract}

Key words and Phrases: Non expansive mapping, Fixed point problem, Projection method, Variational inequality problem, extragradient method.

\section{INTRODUCTION}

Throughout this paper, let $H$ be a real Hilbert Space with norm $\|\cdot\|$ and inner product $\langle\cdot, \cdot\rangle$. Let $D$ be a non empty closed convex subset of $H$. Let $A: D \rightarrow H$ be a non linear mapping then the problem of the variational inequality is to find a point $x \in D$ such that

$$
<A x, y-x>\geq 0, \quad \forall y \in D .
$$

The solution set of the variational inequality is represented by $\Omega$.

A point $x \in D$ is said to be a fixed point if $T x=x$. We adopt $F(T)$ to represent the set of fixed points of $T$. A self mapping $T$ on $D$ is said to be nonexpansive if $\|T x-T y\| \leq\|x-y\|, \forall x, y \in D$.

In 1964, Stampacchia [15] introduced and studied Variational inequality problem. It is notable that a wide classes of issue emerging in pure and applied sciences can be illuminated with the assistance of variational inequality problem or in other words we say that many problems are proved equivalent to variational inequality problem such as optimization problem, maximisation problem. Several researcher

2020 Mathematics Subject Classification: 47H05, 47H09, 47H10.

Received: 11-09-2020, accepted: 15-12-2020. 
works on common solution of the variational inequality and the optimization problem. In fact, there are different approach to study variational inequality problems. Based on distinct approaches, many algorithms for solving variational inequality problem is considered and proposed ( [3], [5], 6], 7], 8], 13], [17, [16]) Let us start with one of the method which is used in our paper, i.e., Korpelevich's extragradient method which was popularized by Korpelevich 9] in 1976 and which initiate a sequence $\left\{a_{n}\right\}$ defined as:

$$
\begin{aligned}
b_{n} & =P_{D}\left(a_{n}-\lambda A a_{n}\right) \\
a_{n+1} & =P_{D}\left(a_{n}-\lambda A b_{n}\right), n \geq 0
\end{aligned}
$$

where $P_{D}$ is the metric projection from $R^{n}$ onto $D, A: D \rightarrow H$ is a monotone operator and $\lambda$ is a constant. Korpelelevich [9] proved that the sequence $\left\{a_{n}\right\}$ converges strongly to a solution of $\mathrm{VI}(\mathrm{D}, \mathrm{A})$.

Korpelevich's extragradient technique has widely been read for the solution of finding common point which belong to the solution set of fixed points of a nonexpansive mapping and variational inequality. In 2006, Nadezhkina and Takahashi [10] introduced the following method:

$$
\begin{aligned}
x_{0} & =x \in D, \\
y_{n} & =P_{D}\left(x_{n}-\lambda_{n} A x_{n}\right), \\
z_{n} & =\alpha_{n} x_{n}+\left(1-\alpha_{n}\right) T P_{D}\left(x_{n}-\lambda_{n} A y_{n}\right), \\
D_{n} & =\left\{z \in D:\left\|z_{n}-z\right\| \leq\left\|x_{n}-z\right\|\right\}, \\
Q_{n} & =\left\{z \in D:<x_{n}-z, x-x_{n}>\geq 0\right\}, \\
x_{n+1} & =P_{D_{n} \cap Q_{n}} x, n \geq 0,
\end{aligned}
$$

where $P_{D}$ is the metric projection from $H$ onto $D$ and $T: D \rightarrow D$ be a nonexpansive mapping with $A: D \rightarrow H$ monotone $k$-Lipschitz continuous mapping having two sequences $\left\{\alpha_{n}\right\}$ and $\left\{\lambda_{n}\right\}$. They established the strong convergence of the sequences, $\left\{x_{n}\right\},\left\{y_{n}\right\}$ and $\left\{z_{n}\right\}$ to the same element of $F(T) \cap \Omega$.

Influenced from the work of Ceng. et al. [1], in which they proved the weak convergence of the iterative method to $\cap_{i=n}^{N} F\left(T_{i}\right) \cap \Omega$ while considering finite family of nonexpansive mapping, Ceng. et al. [2], discovered hybrid extragradient like approximation method for proving strong convergence of this method to $P_{F(T) \cap \Omega}$, Yao. et al. [19], suggested a hybrid method for variational inequality and fixed point of infinite family of nonexpansive mapping and prove its strong convergence to $\cap_{n=i}^{\infty} F\left(T_{n}\right) \cap \Omega$, in this paper, we use hybrid method for finding a typical component of the arrangement of fixed points of an infinite family of nonexpansive mapping and the set of monotone, Lipschtiz continuous variational inequalities problem. The planned method is combination of two methods, one is the extragradient method and the other one is DQ method. Also, we demonstrated the strong convergence of the designed iterative technique, under some warm conditions. 


\section{Preliminaries}

In this section, we recall some basics definitions and lemmas which are further used in our proof. Let D be a nonempty closed convex subset of a real Hilbert space H. A mapping $A: D \rightarrow H$ is called monotone if $\langle A u-A v, u-v\rangle \geq 0, \forall u, v \in D$ and a mapping $T: D \rightarrow D$ is said to be nonexpansive if $\|T x-T y\| \leq\|x-y\|, \forall x, y \in$ $D$. $F(T)$ denote the set of fixed points of $\mathrm{T}$, that is, $F(T)=\{x \in D: T x=x\}$. For every point $x \in H, P_{D}(x)$ represent the unique nearest point in $D$ and $P_{D}$ is called the metric projection of $H$ onto convex subset $D$ and also, a nonexpansive mapping from $H$ onto $D$. It has the following properties:

(i) $P_{D}(x) \in D$

$$
\left\|P_{D}(x)-P_{D}(y)\right\| \leq\|x-y\|, \forall x, y \in H
$$

(ii) $<x-P_{D}(x), y-P_{D}(x)>\leq 0, \forall x \in H, y \in D$

(iii) The property (ii) is equivalent to

$$
\left\|x-P_{D}(x)\right\|^{2}+\left\|y-P_{D}(x)\right\|^{2} \leq\|x-y\|, \forall x \in H, y \in D
$$

(iv) In the variational inequality problem, projection implies that

$$
u \in \Omega, \Longleftrightarrow u=P_{D}(u-\lambda A u), \forall \lambda>0 .
$$

As Opial's condition [11, implies for, any sequence $\left\{x_{n}\right\}$ with $x_{n}$ converges weakly to $x$ and the inequality

$$
\lim _{n \rightarrow \infty} \inf \left\|x_{n}-x\right\|<\lim _{n \rightarrow \infty} \inf \left\|x_{n}-y\right\|
$$

holds $\forall y \in H$ with $y \neq x$.

As $D$ be a nonempty closed and convex subset of $H$. Let $\left\{T_{i}\right\}_{i=1}^{\infty}$ be infinite family of nonexpansive mappings of $D$ into itself and $\left\{\mu_{i}\right\}_{i=1}^{\infty}$ be a real number sequence such that $0 \leq \mu_{i} \leq 1$ for every $i \in N$.

Here, we use the mapping $W_{n}$ [12] defined as

$$
\begin{aligned}
U_{n, n+1} & =I, \\
U_{n, n} & =\mu_{n} T_{n} U_{n, n+1}+\left(1-\mu_{n}\right) I, \\
U_{n, n-1} & =\mu_{n-1} T_{n-1} U_{n, n}+\left(1-\mu_{n-1}\right) I, \\
U_{n, k} & =\mu_{k} T_{k} U_{n, k+1}+\left(1-\mu_{k}\right) I, \\
U_{n, k-1} & =\mu_{k-1} T_{k-1} U_{n, k}+\left(1-\mu_{k-1}\right) I, \\
U_{n, 2} & =\mu_{2} T_{2} U_{n, 3}+\left(1-\mu_{2}\right) I, \\
W_{n} & =U_{n, 1}=\mu_{1} T_{1} U_{n, 2}+\left(1-\mu_{1}\right) I,
\end{aligned}
$$

where $\mu_{1}, \mu_{2}, \cdots$ are real numbers such that $0 \leq \mu_{n} \leq 1$ for each $n \geq 0$ and $T_{1}, T_{2}, \cdots$ are nonexpansive mappings from $D$ into itself. Non expansivity of $T_{i}$ gives us the non expansivity of $W_{n}$. We have the following pivotal lemmas related to $W_{n}[12$ which are stated as: 
Lemma 2.1. 11] Let $D$ be a non empty closed convex subset of real Hilbert space $H$. Let $T_{1}, T_{2}, T_{3}, \cdots$ are nonexpansive mappings of $D$ into itself such that $\cap_{n=1}^{\infty} F\left(T_{n}\right)$ is nonempty. Let $\mu_{1}, \mu_{2}, \mu_{3}, \cdots$ are real numbers such that $0 \leq \mu_{i} \leq 1$ for every $i \in N$. Then for every $x \in D$ and $k \in N$, the limit $\lim _{n \rightarrow \infty} U_{n, k} x$ exists.

Lemma 2.2. 11 Let $D$ be a nonempty closed convex subset of $H$. Let $T_{1}, T_{2}, T_{3}, \cdots$ are nonexpansive mappings of $D$ into itself such that $\cap_{n=1}^{\infty} F\left(T_{n}\right)$ is nonempty. Let $\mu_{1}, \mu_{2}, \mu_{3}, \cdots$ are real numbers such that $0 \leq \mu_{i} \leq 1$ for every $i \in N$. Then, $F(W)=\cap_{n=1}^{\infty} F\left(T_{n}\right)$.

Lemma 2.3. 14 Using the above two lemmas, $W$ is defined from $D$ to itself as: $W x=\lim _{n \rightarrow \infty} W_{n} x=\lim _{n \rightarrow \infty} U_{n, 1} x, \forall x \in D$. If $\left\{x_{n}\right\}$ is bounded sequence in $D$, then

$$
\lim _{n \rightarrow \infty}\left\|W x_{n}-W_{n} x_{n}\right\|=0 .
$$

Lemma 2.4. 18] Let $D$ be a nonempty closed convex subset of $H$. Let $T: D \rightarrow D$ be a nonexpansive mapping with $F(T) \neq \phi$. Then $T$ is demiclosed on $D$, i.e., if $y_{n} \rightarrow z \in D$ weakly and $y_{n}-T y_{n} \rightarrow y$ strongly then $(I-T) z=y$.

Lemma 2.5. 4] Let $D$ be nonempty closed and convex subset of $H$. Let $\left\{x_{n}\right\}$ be a sequence in $H$ and $u \in H$. Let $s=P_{D}(u)$. If $\left\{x_{n}\right\}$ is such that $w_{w}\left(x_{n}\right) \subset D$ and satisfy the equation

$$
\left\|x_{n}-u\right\| \leq\|u-s\|, \forall n
$$

Then $\left\{x_{n}\right\} \rightarrow s$.

Here $w_{w}\left(x_{n}\right)$ respresents the weak convergence of sequence $x_{n}$.

\section{Main Result}

Theorem 3.1. Let $H$ be real Hilbert Space and $D$ be its nonempty closed and convex subset. Let $A: D \rightarrow H$ be a $k$-Lipschitz continuous, monotone, nonexpansive mapping and $\left\{T_{n}\right)_{n=1}^{\infty}$ be an infinite family of nonexpansive mappings of $D$ into itself such that $\cap_{n=1}^{\infty} F\left(T_{n}\right) \cap \Omega \neq \phi$. Let $a_{1}=a_{0} \in D$. For $D_{1}=D$, let $\left\{t_{n}\right\},\left\{c_{n}\right\}$, $\left\{b_{n}\right\}$, and $\left\{a_{n}\right\}$ be sequences generated by:

$$
\begin{aligned}
t_{n} & =P_{D}\left(a_{n}-\lambda_{n} A a_{n}\right) \\
c_{n} & =\beta_{n} a_{n}+\left(1-\beta_{n}\right) W_{n} P_{D}\left(a_{n}-\lambda_{n} A t_{n}\right) \\
b_{n} & =\alpha_{n} a_{n}+\left(1-\alpha_{n}\right) W_{n} P_{D}\left(a_{n}-\lambda_{n} A c_{n}\right), \\
D_{n} & =\left\{s \in D:\left\|b_{n}-s\right\| \leq\left\|a_{n}-s\right\|,\left\|c_{n}-s\right\| \leq\left\|a_{n}-s\right\|\right\}, \\
Q_{n} & =\left\{s \in D:<a_{n}-s, a_{0}-a_{n}>\geq 0\right\}, \\
a_{n+1} & =P_{D_{n} \cap Q_{n}} a_{0}, n \geq 0,
\end{aligned}
$$

where $W_{n}$ is mapping defined above. Assume the following conditions hold:

(i) $\left\{\lambda_{n}\right\} \subset[l, m]$ for some $l, m \in(0,1 / k)$ 
(ii) $\left\{\alpha_{n}\right\},\left\{\beta_{n}\right\} \subset[0, c]$ for some $c \in[0,1)$.

Then the sequences $\left\{t_{n}\right\},\left\{c_{n}\right\},\left\{b_{n}\right\}$, and $\left\{a_{n}\right\}$ converges strongly to the same point $P_{\cap_{n=1}^{\infty} F\left(T_{n}\right) \cap \Omega}\left(a_{0}\right)$.

ProOF. We divide the proof into several parts while proving the main result. We assume that all the assumptions of theorem are satisfied.

Step 1: Firstly, we prove that $D_{n} \cap Q_{n}$ is closed and convex for every $n \in N \cup\{0\}$. As it is obvious that $D_{n}$ is closed and $Q_{n}$ is closed and convex . We only need to prove that $D_{n}$ is convex.

Since, $\left\|b_{n}-s\right\| \leq\left\|a_{n}-s\right\|$ is equivalent to $\left\|b_{n}-a_{n}\right\|^{2}+2<b_{n}-a_{n}, a_{n}-s>\leq 0$ it gives us that $D_{n}$ is convex. Hence, $D_{n} \cap Q_{n}$ is a closed convex subset of $H$ for any $n \in N$

Step 2: We show that $\cap_{n=1}^{\infty} F\left(T_{n}\right) \cap \Omega \subseteq D_{n} \cap Q_{n}$.

For this purpose, set $u_{n}=P_{D}\left(a_{n}-\lambda_{n} A t_{n}\right)$ and $v_{n}=P_{D}\left(a_{n}-\lambda_{n} A c_{n}\right)$ for all $n \geq 1$. Choose $a \in \cap_{n=1}^{\infty} F\left(T_{n}\right) \cap \Omega$. From property (iii) of $P_{D}$, we have

$$
\begin{aligned}
\left\|u_{n}-a\right\|^{2} & \leq\left\|a_{n}-\lambda_{n} t_{n}-a\right\|^{2}-\left\|a_{n}-\lambda_{n} A t_{n}-u_{n}\right\|^{2} \\
& =\left\|a_{n}-a\right\|^{2}-\left\|a_{n}-u_{n}\right\|^{2}+2 \lambda_{n}<A t_{n}, a-u_{n}> \\
& =\left\|a_{n}-a\right\|^{2}-\left\|a_{n}-u_{n}\right\|^{2}+2 \lambda_{n}<A t_{n}, a-t_{n}>+2 \lambda_{n}<A t_{n}, t_{n}-u_{n}>
\end{aligned}
$$

As $a \in \Omega$ and $t_{n} \in D_{n} \subset D$, we get

$$
<A u, t_{n}-a>\geq 0 \text {. }
$$

From the monotonicity of $\mathrm{A}$, we have

$$
<A t_{n}, t_{n}-a>\geq 0 \text {. }
$$

Combining (3) and (4), we get

$$
\begin{aligned}
\left\|u_{n}-a\right\|^{2} & \leq\left\|a_{n}-a\right\|^{2}-\left\|a_{n}-u_{n}\right\|^{2}+2 \lambda_{n}<A t_{n}, t_{n}-u_{n}> \\
& =\left\|a_{n}-a\right\|^{2}-\left\|a_{n}-t_{n}\right\|^{2}-2<a_{n}-t_{n}, t_{n}-u_{n}>-\left\|t_{n}-u_{n}\right\|^{2} \\
& +2 \lambda_{n}<A t_{n}, t_{n}-u_{n}> \\
& =\left\|a_{n}-a\right\|^{2}-\left\|a_{n}-t_{n}\right\|^{2}-\left\|t_{n}-u_{n}\right\|^{2}+2<a_{n}-t_{n}-\lambda_{n} A t_{n}, u_{n}-t_{n}>
\end{aligned}
$$

as $t_{n}=P_{D}\left(a_{n}-\lambda_{n} A a_{n}\right)$ and $u_{n} \in D_{n}$. Then by the property (iii) of $P_{D}$, we have

$$
<a_{n}-\lambda_{n} A a_{n}-t_{n}, u_{n}-t_{n}>\leq 0 .
$$

Hence,

$$
\begin{aligned}
<a_{n}-\lambda_{n} A t_{n}-t_{n}, u_{n}-t_{n}> \\
=<a_{n}-\lambda_{n} A a_{n}-t_{n}, u_{n}-t_{n}>+<\lambda_{n} A a_{n}-\lambda_{n} A t_{n}, u_{n}-t_{n}> \\
\leq<\lambda_{n} A a_{n}-\lambda_{n} A t_{n}, u_{n}-t_{n}><\lambda_{n} k\left\|a_{n}-t_{n}\right\| .\left\|u_{n}-t_{n}\right\| .
\end{aligned}
$$

From (5) and (6), we have

$$
\begin{aligned}
\left\|u_{n}-a\right\|^{2} & \leq\left\|a_{n}-a\right\|^{2}-\left\|a_{n}-t_{n}\right\|^{2}-\left\|t_{n}-u_{n}\right\|^{2}+2<a_{n}-t_{n}-\lambda_{n} A t_{n}, u_{n}-t_{n}> \\
& \leq\left\|a_{n}-a\right\|^{2}-\left\|a_{n}-t_{n}\right\|^{2}-\left\|t_{n}-u_{n}\right\|^{2}+2 \lambda_{n} k\left\|a_{n}-t_{n}\right\| \cdot\left\|u_{n}-t_{n}\right\| \\
& \leq\left\|a_{n}-a\right\|^{2}-\left\|a_{n}-t_{n}\right\|^{2}-\left\|t_{n}-u_{n}\right\|^{2}+\lambda_{n}^{2} k^{2}\left\|a_{n}-t_{n}\right\|^{2}+\left\|u_{n}-t_{n}\right\|^{2} \\
& \leq\left\|a_{n}-a\right\|^{2}+\left(\lambda_{n}^{2} k^{2}-1\right)\left\|a_{n}-t_{n}\right\|^{2} \\
& \leq\left\|a_{n}-a\right\|^{2} .
\end{aligned}
$$


In a similar manner, we can show that

$$
\begin{aligned}
\left\|v_{n}-a\right\|^{2} & \leq\left\|a_{n}-a\right\|^{2}+\left(\left(\lambda_{n}^{2} k^{2}-1\right)\left\|a_{n}-c_{n}\right\|^{2}\right. \\
& \leq\left\|a_{n}-a\right\|^{2} .
\end{aligned}
$$

From (7) together with $c_{n}=\beta_{n} a_{n}+\left(1-\beta_{n}\right) W_{n} u_{n}$ and $a=W_{n} a$, we get

$$
\begin{aligned}
\left\|c_{n}-a\right\|^{2} & =\left\|\beta_{n}\left(a_{n}-a\right)+\left(1-\beta_{n}\right)\left(W_{n} u_{n}-a\right)\right\|^{2} \\
& \leq \beta_{n}\left\|a_{n}-a\right\|^{2}+\left(1-\beta_{n}\right)\left\|W_{n} u_{n}-a\right\|^{2} \\
& \leq \beta_{n}\left\|a_{n}-a\right\|^{2}+\left(1-\beta_{n}\right)\left\|u_{n}-a\right\|^{2} \\
& \leq \beta_{n}\left\|a_{n}-a\right\|^{2}+\left(1-\beta_{n}\right)\left(\left\|a_{n}-a\right\|^{2}+\left(\lambda_{n}^{2} k^{2}-1\right)\left\|a_{n}-u_{n}\right\|^{2}\right) \\
& \leq\left\|a_{n}-a\right\|^{2}+\left(1-\beta_{n}\right)\left(\lambda_{n}^{2} k^{2}-1\right)\left\|a_{n}-u_{n}\right\|^{2} \\
& \leq\left\|a_{n}-a\right\|^{2} .
\end{aligned}
$$

Again, from (8) together with $b_{n}=\alpha_{n} a_{n}+\left(1-\alpha_{n}\right) W_{n} v_{n}$ and $a=W_{n} a$, we obtain

$$
\begin{aligned}
\left\|b_{n}-a\right\|^{2} & =\left\|\alpha_{n}\left(a_{n}-a\right)+\left(1-\alpha_{n}\right)\left(W_{n} v_{n}-a\right)\right\|^{2} \\
& \leq\left\|a_{n}-a\right\|^{2}+\left(1-\alpha_{n}\right)\left(\lambda_{n}^{2} k^{2}-1\right)\left\|a_{n}-c_{n}\right\|^{2} \\
& \leq\left\|a_{n}-a\right\|^{2} .
\end{aligned}
$$

Thus, $a \in D_{n}$ for $\left\{b_{n}\right\}$.

From, $(9)$ and $(10)$, we get

$$
a \in D_{n}
$$

and hence

$$
\cap_{n=1}^{\infty} F\left(T_{n}\right) \cap \Omega \subseteq D_{n} \forall n \in N .
$$

Next, we show that

$$
\cap_{n=1}^{\infty} F\left(T_{n}\right) \cap \Omega \subset D_{n} \cap Q_{n} \forall n \in N .
$$

We prove this by induction. For $n=1$, we have

$$
\cap_{n=1}^{\infty} F\left(T_{n}\right) \cap \Omega \subset D_{1} \text { and } Q_{1}=H,
$$

and we get

$$
\cap_{n=1}^{\infty} F\left(T_{n}\right) \cap \Omega \subset D_{1} \cap Q_{1} .
$$

Assume $a_{k}$ is defined and $\cap_{n=1}^{\infty} F\left(T_{n}\right) \cap \Omega \subset D_{k} \cap Q_{k}$ for some $k \geq 0$. Then $D_{k}$ and $Q_{k}$ is closed and convex due to well defined nature of $c_{k}$ and $b_{k}$ as elements of $D$. Thus $D_{k} \cap Q_{k}$ is closed and convex subset, which is nonempty since by assumption of $\cap_{n=1}^{\infty} F\left(T_{n}\right) \cap \Omega$. Consequently, $a_{n+1} \in D_{n} \cap Q_{n}$ such that

$$
a_{n+1}=P_{D_{n} \cap Q_{n}} a_{0},
$$


then

$$
<a_{n+1}-z, a_{0}-a_{n+1}>\geq 0 \text { for each } z \in D_{n} \cap Q_{n} .
$$

As especial

$$
<a_{n+1}-z, a_{0}-a_{n+1}>\geq 0 \text { for each } z \in \cap_{n=1}^{\infty} F\left(S_{n}\right) \cap \Omega
$$

and hence $z \in Q_{n+1}$. It follows that $\cap_{n=1}^{\infty} F\left(T_{n}\right) \cap \Omega \subset Q_{n+1}$. This together with (11) gives

$$
\cap_{n=1}^{\infty} F\left(T_{n}\right) \cap \Omega \subset D_{n} \cap Q_{n}, \forall n .
$$

Thus $\left\{a_{n}\right\}$ is well-defined.

Step 3: Now, we show that $\left\{a_{n}\right\}$ is bounded.

Since $\cap_{n=1}^{\infty} F\left(T_{n}\right) \cap \Omega$ is a nonempty closed convex subset of $\mathrm{H}$, then there exist a unique $z_{0} \in \cap_{n=1}^{\infty} F\left(T_{n}\right) \cap \Omega$ such that $z_{0}=P_{\cap_{n=1}^{\infty} F\left(T_{n}\right) \cap \Omega} a_{0}$. From $a_{n+1}=$ $P_{D_{n} \cap Q_{n}} a_{0}$, we have

$$
\left\|a_{n+1}-a_{0}\right\| \leq\left\|z-a_{0}\right\| \text { for every } z \in D_{n} \cap Q_{n} \text { and for every } n \in N \text {. }
$$

Since $z_{0} \in \cap_{n=1}^{\infty} F\left(T_{n}\right) \cap \Omega \subset D_{n} \cap Q_{n}$, we have

$$
\left\|a_{n+1}-a_{0}\right\| \leq\left\|z_{0}-a_{0}\right\|, \forall n \in N \text {. }
$$

Thus, we obtain $\left\{a_{n}\right\}$ is bounded.

As $a_{n+1} \in D_{n} \cap Q_{n}$ and $a_{n}=P_{Q_{n}} a_{0}$, we have

$$
\begin{aligned}
& <a_{0}-a_{n}, a_{n}-a_{n+1}>\geq 0 \\
& 0 \leq-\left\|a_{0}-a_{n}\right\|^{2}+\left\|a_{0}-a_{n}\right\| \cdot\left\|a_{0}-a_{n+1}\right\|,
\end{aligned}
$$

and therefore

$$
\left\|a_{n+1}-a_{0}\right\| \geq\left\|a_{n}-a_{0}\right\| .
$$

This together with the boundedness of the sequence $\left\{a_{n}\right\}$ imply that $\lim _{n \rightarrow \infty}\left\|a_{n}-a_{0}\right\|$ exists.

Step 4: Now, we obtain the following equalities

$$
\lim _{n \rightarrow \infty}\left\|a_{n+1}-a_{n}\right\|=\lim _{n \rightarrow \infty}\left\|a_{n}-c_{n}\right\|=\lim _{n \rightarrow \infty}\left\|a_{n}-t_{n}\right\|=0
$$

and

$$
\lim _{n \rightarrow \infty}\left\|a_{n}-W_{n} a_{n}\right\|=\lim _{n \rightarrow \infty}\left\|b_{n}-W_{n} b_{n}\right\|=0 .
$$

Consider

$$
\begin{aligned}
\left\|a_{n+1}-a_{n}\right\|^{2} & =\left\|\left(a_{n+1}-a_{0}\right)-\left(a_{n}-a_{0}\right)\right\|^{2} \\
& =\left\|a_{n+1}-a_{0}\right\|^{2}-\left\|a_{n}-a_{0}\right\|^{2}-2<a_{n+1}-a_{0}, a_{n}-a_{0}> \\
& \leq\left\|a_{n+1}-a_{0}\right\|^{2}-\left\|a_{n}-a_{0}\right\|^{2} .
\end{aligned}
$$

As $\lim _{n \rightarrow \infty}\left\|a_{n}-a_{0}\right\|$ exists, we get

$$
\left\|a_{n+1}-a_{0}\right\|^{2}-\left\|a_{n}-a_{0}\right\|^{2} \rightarrow 0 .
$$


Therefore, $\lim _{n \rightarrow \infty}\left\|a_{n+1}-a_{n}\right\|=0$.

Since $a_{n+1} \in D_{n}$, we have

$$
\left\|b_{n}-a_{n+1}\right\| \leq\left\|a_{n}-a_{n+1}\right\|
$$

and

$$
\begin{aligned}
\left\|a_{n}-b_{n}\right\| & \leq\left\|a_{n}-a_{n+1}\right\|+\left\|a_{n+1}-b_{n}\right\| \\
& \leq 2\left\|a_{n+1}-a_{n}\right\| \rightarrow 0 .
\end{aligned}
$$

Similarly, we get $\left\|a_{n}-c_{n}\right\| \rightarrow 0$.

Now, for each $u \in \cap_{n=1}^{\infty} F\left(T_{n}\right) \cap \Omega$ from $(8)$, we have

$$
\begin{aligned}
\left\|a_{n}-t_{n}\right\|^{2} & \leq \frac{1}{\left(1-\beta_{n}\right)\left(\lambda_{n}^{2} k^{2}-1\right)}\left(\left\|a_{n}-a\right\|^{2}-\left\|c_{n}-a\right\|^{2}\right) \\
& \leq \frac{1}{\left(1-\beta_{n}\right)\left(\lambda_{n}^{2} k^{2}-1\right)}\left(\left\|a_{n}-a\right\|+\left\|c_{n}-a\right\|\right)\left(\left\|a_{n}-c_{n}\right\|\right) .
\end{aligned}
$$

Since $\left\|a_{n}-c_{n}\right\| \rightarrow 0$, sequence $\left\{a_{n}\right\}$ and $\left\{c_{n}\right\}$ are bounded, we obtain

$$
\left\|a_{n}-t_{n}\right\| \rightarrow 0 \text {. }
$$

By the same idea as in (7), we obtain that

$$
\left\|u_{n}-a\right\|^{2} \leq\left\|a_{n}-a\right\|^{2}+\left(\lambda_{n}^{2} k^{2}-1\right)\left\|t_{n}-u_{n}\right\|^{2} .
$$

Hence,

$$
\begin{aligned}
\left\|c_{n}-a\right\|^{2} & \leq \beta_{n}\left\|a_{n}-a\right\|^{2}+\left(1-\beta_{n}\right)\left\|u_{n}-a\right\|^{2} \\
& \leq \beta_{n}\left\|a_{n}-a\right\|^{2}+\left(1-\beta_{n}\right)\left(\left\|a_{n}-a\right\|^{2}+\left(\lambda_{n}^{2} k^{2}-1\right)\left\|t_{n}-u_{n}\right\|^{2}\right) \\
& =\left\|a_{n}-a\right\|^{2}+\left(1-\beta_{n}\right)\left(\lambda_{n}^{2} k^{2}-1\right)\left\|t_{n}-u_{n}\right\|^{2} .
\end{aligned}
$$

It follows that

$$
\begin{aligned}
\left\|t_{n}-u_{n}\right\|^{2} & \leq \frac{1}{\left(1-\beta_{n}\right)\left(\lambda_{n}^{2} k^{2}-1\right)}\left(\left\|a_{n}-a\right\|-\left\|c_{n}-a\right\|\right)\left\|a_{n}-c_{n}\right\| \\
& \rightarrow 0
\end{aligned}
$$

From the k-Lipschitz continuity of $\mathrm{A}$, we have $\left\|A b_{n}-A t_{n}\right\| \rightarrow 0$, from

$$
\left\|a_{n}-u_{n}\right\| \leq\left\|a_{n}-t_{n}\right\|+\left\|t_{n}-u_{n}\right\|,
$$

we get

$$
\left\|a_{n}-u_{n}\right\| \rightarrow 0 \text {. }
$$

In a similar manner and from the idea as in (7), we get

$$
\left\|v_{n}-a\right\|^{2} \leq\left\|a_{n}-a\right\|^{2}+\left(\lambda_{n}^{2} k^{2}-1\right)\left\|t_{n}-v_{n}\right\| .
$$


Hence,

$$
\begin{aligned}
\left\|b_{n}-a\right\|^{2} & \leq \alpha_{n}\left\|a_{n}-a\right\|^{2}+\left(1-\alpha_{n}\right)\left(\left\|a_{n}-a\right\|^{2}+\left(\lambda_{n}^{2} k^{2}-1\right)\left\|t_{n}-v_{n}\right\|^{2}\right) \\
& \leq\left\|a_{n}-a\right\|^{2}+\left(1-\alpha_{n}\right)\left(\lambda_{n}^{2} k_{2}-1\right)\left\|t_{n}-v_{n}\right\|^{2} .
\end{aligned}
$$

It follows that

$$
\begin{aligned}
\left\|t_{n}-v_{n}\right\|^{2} & \leq \frac{1}{\left(1-\alpha_{n}\right)\left(\lambda_{n}^{2} k^{2}-1\right)}\left(\left\|a_{n}-a\right\|^{2}-\left\|b_{n}-a\right\|^{2}\right) \\
& \leq \frac{1}{\left(1-\alpha_{n}\right)\left(\lambda_{n}^{2} k^{2}-1\right)}\left(\left\|a_{n}-a\right\|+\left\|b_{n}-a\right\|\right)\left\|a_{n}-b_{n}\right\| \\
& \rightarrow 0,
\end{aligned}
$$

and from

$$
\left\|a_{n}-v_{n}\right\| \leq\left\|a_{n}-b_{n}\right\|+\left\|b_{n}-v_{n}\right\|,
$$

we have

$$
\left\|a_{n}-v_{n}\right\| \rightarrow 0 \text {. }
$$

Since $c_{n}=\beta_{n} a_{n}+\left(1-\beta_{n}\right) W_{n} u_{n}$, we find

$$
\left(1-\beta_{n}\right)\left(W_{n} u_{n}-u_{n}\right)=\beta_{n}\left(u_{n}-a_{n}\right)+\left(c_{n}-u_{n}\right)
$$

and

$$
\begin{aligned}
(1-c)\left\|W_{n} u_{n}-u_{n}\right\| & \leq\left(1-\beta_{n}\right)\left\|W_{n} u_{n}-u_{n}\right\| \\
& \leq \beta_{n}\left\|u_{n}-a_{n}\right\|+\left\|c_{n}-u_{n}\right\| \\
& \leq\left(1+\beta_{n}\right)\left\|u_{n}-a_{n}\right\|+\left\|c_{n}-a_{n}\right\| .
\end{aligned}
$$

Hence,

$$
\left\|u_{n}-W_{n} u_{n}\right\| \rightarrow 0 .
$$

In a similar way, as

$$
b_{n}=\alpha_{n} a_{n}+\left(1-\alpha_{n}\right) v_{n},
$$

we have

$$
\left(1-\alpha_{n}\right)\left(W_{n} v_{n}\right)=\alpha_{n}\left(v_{n}-a_{n}\right)+\left(b_{n}-v_{n}\right)
$$

and

$$
\begin{aligned}
(1-c)\left\|W_{n} v_{n}-v_{n}\right\| & \leq\left(1-\alpha_{n}\right)\left\|W_{n} v_{n}-v_{n}\right\| \\
& \leq \alpha_{n}\left\|v_{n}-a_{n}\right\|+\left\|b_{n}-a_{n}\right\| \\
& \leq\left(1+\alpha_{n}\right)\left\|v_{n}-a_{n}\right\|+\left\|b_{n}-a_{n}\right\| .
\end{aligned}
$$


Hence,

$$
\left\|v_{n}-W_{n} v_{n}\right\| \rightarrow 0
$$

To conclude

$$
\begin{aligned}
\left\|a_{n}-W_{n} a_{n}\right\| & \leq\left\|a_{n}-u_{n}\right\|+\left\|u_{n}-W_{n} u_{n}\right\|+\left\|W_{n} u_{n}-W_{n} a_{n}\right\| \\
& \leq\left\|a_{n}-u_{n}\right\|+\left\|u_{n}-W_{n} u_{n}\right\|+\left\|u_{n}-a_{n}\right\| \\
& \leq 2\left\|a_{n}-u_{n}\right\|+\left\|u_{n}-W_{n} u_{n}\right\|
\end{aligned}
$$

So

$$
\left\|a_{n}-W_{n} a_{n}\right\| \rightarrow 0 .
$$

Similarly, from 15), we get

$$
\left\|b_{n}-W_{n} b_{n}\right\| \rightarrow 0 .
$$

As, $\left\|a_{n}-W_{n} a_{n}\right\|$ and $\left\|b_{n}-W_{n} b_{n}\right\| \rightarrow 0$

On the other hand, since $\left\{a_{n}\right\}$ and $\left\{b_{n}\right\}$ are bounded and from lemma 2.3), we have

$$
\lim _{n \rightarrow \infty}\left\|W_{n} a_{n}-W a_{n}\right\|=0
$$

and

$$
\lim _{n \rightarrow \infty}\left\|W_{n} b_{n}-W b_{n}\right\|=0
$$

Therefore, we have

$$
\lim _{n \rightarrow \infty}\left\|a_{n}-W a_{n}\right\|=0
$$

and

$$
\lim _{n \rightarrow \infty}\left\|b_{n}-W b_{n}\right\|=0 .
$$

Step 5: Strong convergence of $\left\{t_{n}\right\},\left\{b_{n}\right\},\left\{c_{n}\right\}$ and $\left\{a_{n}\right\}$ to $P_{\cap_{n=1}^{\infty} F\left(T_{n}\right) \cap \Omega} a_{0}$. Furthermore, since $\left\{a_{n}\right\}$ and $\left\{b_{n}\right\}$ is bounded and has a subsequence $\left\{a_{n_{i}}\right\}$ and $\left\{b_{n_{i}}\right\}$ which converges weakly to some $a \in D$, hence we have

$$
\lim _{j \rightarrow \infty}\left\|a_{n_{j}}-W a_{n_{j}}\right\|=0 \text { and } \lim _{j \rightarrow \infty}\left\|b_{n_{j}}-W b_{n_{j}}\right\|=0 .
$$

From lemma (2.4), which gives that $I-W$ is demiclosed at zero. Thus, $a \in F(W)$. Since $u_{n}=P_{D_{n}}\left(a_{n}-\lambda_{n} A t_{n}\right)$ and $v_{n}=P_{D_{n}}\left(a_{n}-\lambda_{n} A c_{n}\right)$, for every $x \in D_{n}$, we have

$$
<a_{n}-\lambda_{n} A t_{n}-u_{n}, u_{n}-x>\geq 0 \text { and }<a_{n}-\lambda_{n} A c_{n}-v_{n}, v_{n}-x>\geq 0
$$

hence,

$$
<x-u_{n}, A t_{n}>\geq<x-u_{n}, \frac{a_{n}-u_{n}}{\lambda_{n}}>\text { and }<x-v_{n}, A c_{n}>\geq<x-v_{n}, \frac{a_{n}-v_{n}}{\lambda_{n}}>
$$


Combinig with monotonicity of $\mathrm{A}$ and consider $s_{n}=u_{n}+v_{n}$, we have

$$
\begin{aligned}
<x-s_{n}, A x>= & <x-\left(u_{n}+v_{n}\right), A x> \\
= & <x-u_{n}, A x>+<x-v_{n}, A x> \\
\geq & <x-u_{n}, A u_{n}>+<x-v_{n}, A v_{n}> \\
\geq & <x-u_{n}, A u_{n}-A c_{n}>+<x-u_{n}, A c_{n}> \\
& +<x-v_{n}, A v_{n}-A b_{n}>+<x-v_{n}, A b_{n}> \\
\geq & <x-u_{n}, A u_{n}-A c_{n}>+<x-v_{n}, A v_{n}-A b_{n}> \\
& +<x-u_{n}, \frac{a_{n}-u_{n}}{\lambda_{n}}>+<x-v_{n}, \frac{a_{n}-v_{n}}{\lambda_{n}}>
\end{aligned}
$$

Since $\lim _{n \rightarrow \infty}\left(a_{n}-u_{n}\right)=\lim _{n \rightarrow \infty}\left(c_{n}-u_{n}\right)=0$ and $\lim _{n \rightarrow \infty}\left(a_{n}-v_{n}\right)=\lim _{n \rightarrow \infty}\left(b_{n}-\right.$ $\left.v_{n}\right)=0, \mathrm{~A}$ is Lipschitz continuous and $\lambda_{n} \geq l>0$, we deduce that

$$
<x-a, A x>=\lim _{n_{i} \rightarrow \infty}<x-s_{n_{i}}, A x>\geq 0
$$

This implies that $a \in \Omega$. Consequently, $a \in \cap_{n=1}^{\infty} F\left(T_{n}\right) \cap \Omega$ that is $w_{w}\left(a_{n}\right) \subset \cap_{n=1}^{\infty} F\left(T_{n}\right) \cap \Omega$.

In 12 , if we assume $u=P_{\cap_{n=1}^{\infty} F\left(T_{n}\right) \cap \Omega} a_{0}$, we get

$$
\left\|a_{0}-a_{n+1}\right\| \leq\left\|a_{0}-P_{\cap_{n=1}^{\infty} F\left(T_{n}\right) \cap \Omega} a_{0}\right\|
$$

Notice that $w_{w}\left(a_{n}\right) \subset \cap_{n=1}^{\infty} F\left(T_{n}\right) \cap \Omega$. Then, (16) and lemma 2.5 ensure the strong convergence of $\left\{a_{n+1}\right\}$ to $P_{\cap_{n=1}^{\infty} F\left(T_{n}\right) \cap \Omega} a_{0}$.

Consequently, $\left\{t_{n}\right\},\left\{b_{n}\right\}$ and $\left\{c_{n}\right\}$ also converges strongly to $P_{\cap_{n=1}^{\infty} F\left(T_{n}\right) \cap \Omega} a_{0}$.

Hence the result.

Remark 1: We obtain the result of [9], if infinite family of mappings reduces to single mapping with $\beta_{n}=0$ and $W_{n}=I$.

Remark 2: If $Q_{n}=0$ and equation (2) reduces to two step iteration, then we obtain Theorem 3.1 of [19].

\section{Numerical Example}

In this part, we give an example which supports our result.

Example: Let $H=R$ and $D=[0,2]$. Let $\alpha_{n}=n /(n+2), \beta_{n}=n /(n+3)$, $\lambda_{n}=1+1 / n, A x=1 / 3(x-1)$ and $W_{n}(x)=2 x / n$.

For $\left\{a_{n}\right\}$ defined in (2), we divide this procedure into 3 steps:

Setp 1: Find $D_{n}$. Since $D_{n}=\left\{s \in D:\left\|b_{n}-s\right\| \leq\left\|a_{n}-s\right\|,\left\|c_{n}-s\right\| \leq\left\|a_{n}-s\right\|\right\}$, we obtain $\left(2 s-\left(a_{n}+b_{n}\right)\right)\left(a_{n}-b_{n}\right) \leq 0$ and $\left(2 s-\left(a_{n}+c_{n}\right)\right)\left(a_{n}-c_{n}\right) \leq 0$. We have the different cases:

Case1: If $a_{n}-b_{n}=0$, then $D_{n}=D \forall n \geq 1$.

Case 2: If $a_{n}-b_{n}>0$ then $s \leq \frac{a-n+b_{n}}{2}$. Thus $D_{n}=\left[0, \frac{a_{n}+b_{n}}{2}\right], \forall n \geq 1$.

Case 3: If $a_{n}-b_{n}<0$ then $s \geq \frac{a-n+b_{n}}{2}$. Thus $D_{n}=\left[\frac{a_{n}+b_{n}^{2}}{2}, 2\right], \forall n \geq 1$.

Similarly, we have $D_{n}=\left[0, \frac{a_{n}+c_{n}}{2}\right]$ for $a_{n}-c_{n}>0$ and $D_{n}=\left[\frac{a_{n}+c_{n}}{2}, 2\right]$ for $a_{n}-c_{n}<$ $0 \forall n \geq 1$. 
Thus $D_{n}$ is intersection of possible cases generated due to $\left\{b_{n}\right\}$ and $\left\{c_{n}\right\}$.

Step 2: Find $Q_{n}=\left\{s \in Q:<a_{n}-s, x-a_{n}>\geq 0\right\}=\left\{s \in Q:\left(a_{n}-s\right)\left(x-a_{n}\right) \geq 0\right\}$. We obtain the following cases:

Case 1: If $x-a_{n}=0 \Rightarrow Q_{n}=D$.

Case 2: $x-a_{n}>0 \rightarrow a_{n}-s \geq 0 \Rightarrow s \leq a_{n} \Rightarrow Q_{n}=D \cap\left[0, a_{n}\right]$

Case 3: $x-a_{n}<0 \rightarrow a_{n}-s \leq 0 \Rightarrow s \geq a_{n} \Rightarrow Q_{n}=D \cap\left[a_{n}, 2\right]$.

Step 3: Calculate the numerical result of $a_{n+1}=P_{D_{n} \cap Q_{n}} a_{1}$. Take $a_{1}=0.19$, we obtain the Table 1 and we observe that 0 is the solution of our iteration.

TABLE 1. Numerical result of iteration when $a_{1}=0.19$

\begin{tabular}{ccccccccc}
\hline $\mathrm{n}$ & $\lambda_{n}$ & $\alpha_{n}$ & $\beta_{n}$ & $t_{n}$ & $c_{n}$ & $b_{n}$ & $D_{n} \cap Q_{n}$ & $a_{n}$ \\
\hline 1 & 2 & 0.333 & 0.25 & 0.73 & 0.6025 & 0.67 & {$[0.24,2]$} & 0.19 \\
2 & 1.5 & 0.5 & 0.4 & 0.62 & 0.354 & 0.4015 & {$[0.08075,2]$} & 0.24 \\
3 & 1.33 & 0.6 & 0.5 & 0.489 & 0.143 & 0.172 & {$[0.0454,0.10925]$} & 0.08075 \\
4 & 1.25 & 0.67 & 0.5714 & 0.443 & 0.085 & 0.101 & {$[0.028,0.145]$} & 0.0454 \\
5 & 1.2 & 0.714 & 0.625 & 0.417 & 0.0567 & 0.066 & {$[0.0192,0.162]$} & 0.028 \\
6 & 1.167 & 0.75 & 0.67 & 0.4006 & 0.0408 & 0.047 & {$[0.0139,0.171]$} & 0.019 \\
12 & 1.083 & 0.852 & 0.8 & 0.364 & 0.012 & 0.013 & {$[0.0039,0.1853]$} & 0.005 \\
16 & 1.0625 & 0.89 & 0.84 & 0.35 & 0.006 & 0.007 & {$[0.0023,0.187]$} & 0.002 \\
19 & 1.053 & 0.904 & 0.86 & 0.352 & 0.0048 & 0.0052 & {$[0.0017,0.188]$} & 0.0018 \\
$\vdots$ & $\vdots$ & $\vdots$ & $\vdots$ & $\vdots$ & $\vdots$ & $\vdots$ & $\vdots$ & $\vdots$ \\
28 & 1.035 & 0.93 & 0.903 & 0.346 & 0.00234 & 0.00244 & {$[0.00079,0.00081]$} & 0.00086 \\
29 & 1.034 & 0.935 & 0.906 & 0.345 & 0.00218 & 0.0022 & {$[0.00058,0.00066]$} & 0.00079 \\
\hline
\end{tabular}

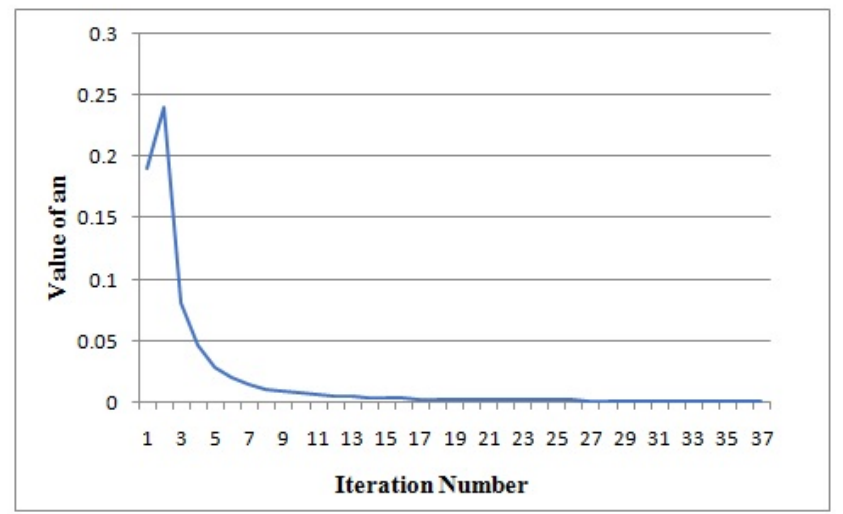

Figure 1. Convergence of $\left\{a_{n}\right\}$

\section{Conclusion}

In this paper, we proposed an algorithm to obtain its strong convergence and calculate the common solution of the infinite family of nonexpansive mappings with 
the variational inequality problem under some imposed conditions over algorithm. The efficiency of the proposed algorithm has also been illustrated by numerical example.

\section{REFERENCES}

[1] Ceng, L. C, Teboulle, M. and Yao, J. C., " Weak convergence of an iterative method for pseudo-monotone variational inequalities and fixed point problems", J Optim Theory Appl. 146(2010),19-31.

[2] Ceng, L. C., Hadjisavvas, N. and Wong, N. C., "Strong convergence theorem by a hybrid extragradient-like approximation method for variational inequalities and fixed point problems", J. Glob Optim., 46(2010), 635-646.

[3] Cholamjiak, P., Thong D.V. and Cho, Y.J., "A novel inertial projection and contraction method for solving pseudomonotone variational inequality problems", Acta Appl. Math. 169(2019), 217-245.

[4] Goebel, K. and Kirk, W. A., " Topics in Metric Fixed Point Theory", Cambridge Studies in Advanced Mathematics, Cambridge University Press, Cambridge, 28(1990).

[5] Hieu, D. V., "Parallel and cyclic hybrid subgradient extragradient methods for variational inequalities, Afrika Matematika, 28(2017), 677-692.

[6] Hieu, D.V. and Cholamjiak, P., "Modified extragradient method with Bregman distance for variational inequalities", Applicable Analysis, (2020), 16 pages.

[7] Hammed, H. A., ur Rehman, H. and De la Sen, M., "Advanced algorithms and common solutions to variational inequalities, Symmetry-Basel, 12(7)(2020), 1198.

[8] Kesornprom, S. and Cholamjiak, P., "Proximal type algorithms involving linesearch and inertial technique for split variational inclusion problem in hilbert spaces with applications", Optimization, 68(2019), 2365-2391.

[9] Korpelevich, G. M. "An extragradient method for finding saddle points and other problems", Ekonomikai Matematicheskie Metody, 12(1976), 747-756.

[10] Nadezhkina, N., Takahashi, W., "Strong convergence theorem by a hybrid method for nonexpansive mappings andLipschitz-continuous monotone mappings", SIAM J Optim., 16(2006), 1230-1241.

[11] Opial, Z., "Weak convergence of the sequence of successive approximations of nonexpansive mappings, Bull. Am. Math. Soc., 73(1976), 595-597.

[12] Qin, X., Cho, Y. J. Kang, J. I. and Kang, S. M., "Strong convergence theorems for an infinte family of nonexpansive mappings in Banach spaces", J.of Computational and Applied Mathematics, 230(2009), 121-127.

[13] Shehu, Y. and Cholamjiak, P., "Iterative method with inertial for variational inequalities in Hilbert spaces", Calcolo, 56(2019), 21 pages.

[14] Shimoji, K. and Takahashi, W., "Strong convergence to common fixed points of infinite nonexpasnsive mappings andapplications" Taiwan J. Math.,5(2001), 387-404.

[15] Stampacchia, G., "Formes bilinearies coercitives sur les ensembles convexes", D.R. Math. Sci. Paris., 258(1964), 4414-4416.

[16] Suantai, S., Kesornprom, S. and Cholamjiak, P., "Modified proximal algorithms for finding solutions of the split variational inclusions", Mathematics, 7(8)(2019), 708.

[17] Yang, J. and Liu, H., "The subgradient extragradient pseudomonotone equilibrium problems and fixed point problems in Hilbert space, Optimization Letters, 14(1)(2020), 1803-1816.

[18] Yao, Y., Liou, Y. C. and Yao, J. C., " Convergence theorem for equilibrium problems and fixed point problems of infinite familyof nonexpansive mappings", Fixed Point Theory and Applications, 12 (2007).

[19] Yao, Y., Liou, Y. C., Wong, M. and Yao, J. C., " Strong convergence of a hybrid method for monotone variational inequalities and fixed point problems", Fixed Point Theory and Applications, 1-10(2011). 